innervation to the hippocampus is critical for normal learning and memory and is severely degenerated in Alzheimer's disease. To understand the molecular events underlying this loss, we optimized a primary septal-hippocampal co-culture system that facilitates the study of central cholinergic synapses. METHODS/STUDY POPULATION: We developed an optimized in vitro septal-hippocampal co-culture system modified from previously published protocols. Briefly, hippocampal and septal tissue were harvested from embryonic day 19 (E19) Sprague-Dawley rats, digested with $0.1 \%$ trypsin, and an equal number of cells from each region plated onto coverslips coated with poly-D-lysine and laminin at a final density of 300 cells $/ \mathrm{mm} 2$. We use immunostaining with validated primary antibodies and a fluorescent binding assay, together with confocal microscopy, to determine the structure of cholinergic synapses that are 1) native, 2) mammalian, 3) CNS derived, 4) comprised of physiological synaptic partners, and 5) developmentally mature. RESULTS/ANTICIPATED RESULTS: After DIV21, co-cultures maintained a healthy morphology. A subpopulation of neurons strongly expressed the cholinergic markers vesicular ACh transporter (vAChT), choline acetyltransferase (ChAT), and the highaffinity choline transporter (ChT1), whereas most neurons lacked vAChT expression and were presumably glutamatergic or GABAergic. The percentage of cholinergic neurons attained in the co-culture is $\sim 5-7 \%$. The size of these cholinergic neurons is strikingly similar to that reported for BFCNs in the intact brain (mean $30 \mu \mathrm{m}$, range $18-43 \mu \mathrm{m}$ ). All sampled cholinergic neurons (28/28 neurons) also expressed molecular machinery necessary for GABA release. Staining for a cholinergic postsynaptic marker shows that $63 \%$ of the contacts made with are synaptic. DISCUSSION/ SIGNIFICANCE OF FINDINGS: Primary septal-hippocampal cocultured neurons have not been exploited extensively in the field, perhaps due to the difficulty in maintaining such cultures for extended periods. Here, we optimized an in vitro septal-hippocampal co-culture system, a powerful tool to comprehensively analyze central cholinergic synapse formation and dysfunction.

\section{Data Science/Biostatistics/Informatics}

23255

\section{Devices Engineered to Collect Exhaled Breath Condensate (EBC) and their Applications*}

Alexander J. Schmidt ${ }^{1}$, Eva Borras ${ }^{1}$, Anh Nguyen ${ }^{2}$, Nicholas J. Kenyon $^{2}$ and Cristina E. Davis ${ }^{1}$

${ }^{1}$ Department of Mechanical and Aerospace Engineering, University of California, Davis and ${ }^{2}$ Department of Internal Medicine, University of California, Davis

ABSTRACT IMPACT: Human exhaled breath is rich in metabolomic content that represents pulmonary function and gas exchange with blood, which can provide insights into an individual's state of health. OBJECTIVES/GOALS: Human exhaled breath is rich in metabolomic content that represents pulmonary function and gas exchange with blood. It contains a mixture of compounds that offer insight into an individual's state of health. Here, we present two novel non-invasive breath sampling devices for use in basic medical practice. METHODS/STUDY POPULATION: The two breath samplers have a disposable mouthpiece, a set of inhale and exhale one-way flap valves to allow condensation of exhaled breath only, and a saliva filter. The housing is constructed out of Teflon ${ }^{\circledR}$, a chemically inert material to reduce chemical absorbance. The first device condenses exhaled breath into a frozen condensate using dry ice pellets and the other is a miniaturized design that liquifies exhaled breath on a condenser surface with micropatterned features on a cooling plate. Both designs have individual strategic and analytical advantages: frozen exhaled breath condensate (EBC) has high retention of analytes and sample volume; EBC collected in liquid phase offers facilitated sample collection and device portability. RESULTS/ANTICIPATED RESULTS: We investigated if breath aerosol size distribution affects the types or abundances of metabolites. We modified the geometry of the first device to redirect aerosol trajectories based on size. The trapping of larger aerosols increases with filter length, thus altering the aerosol size distribution although no significant changes in the metabolite profiles were found. With the miniaturized device, metabolite abundances were measured in a small cohort of healthy control and mild asthmatic subjects. Differences among subjects were found, as well as main differences between control and asthmatic groups. All analyses of EBC were performed with liquid chromatography - mass spectrometry. Inflammatory suppression found in asthmatic subjects can be explained by prescribed daily use of inhaled corticosteroids. DISCUSSION/SIGNIFICANCE OF FINDINGS: Breath collection devices can be used in intensive care units, outpatient clinics, workplaces, and at home. EBC analysis has been used to monitor asthma and chronic obstructive pulmonary disease. It can be applied to infectious respiratory diseases (e.g. influenza, COVID-19) and for monitoring environmental and occupational chemical exposures.

30432

Novel insights from single-cell RNAseq analysis of the stromavascular fraction of human adipose tissue Bingyao Wang ${ }^{1}$, Gregory Smith ${ }^{2}$, Frederique Ruf-Zamojski ${ }^{2}$, Gyu Ho Lee $^{3}$, Stuart C. Sealfon ${ }^{2}$, Jeanine Albu ${ }^{2,4}$, Susan K. Fried ${ }^{3}$ and Kalypso Karastergiou ${ }^{4}$

${ }^{1}$ Icahn School of Medicine at Mount Sinai, ${ }^{2}$ Department of Neurology at Icahn School of Medicine at Mount Sinai, ${ }^{3}$ Diabetes, Obesity and Metabolism Institute at Icahn School of Medicine at Mount Sinai and ${ }^{4}$ Obesity and Metabolism Institute at Icahn School of Medicine at Mount Sinai

ABSTRACT IMPACT: Characterizing the cellular composition of human adipose tissue may contribute to the prevention and/or treatment of obesity-associated metabolic diseases. OBJECTIVES/ GOALS: Our aims in this study were to use single-cell techniques 1. to characterize cell types within the stromavascular fraction of human adipose tissue, 2 . to identify subsets of cells within each type (sub-clustering), 3. to identify gene sets and pathways that may provide information on the function and significance of each cell cluster. METHODS/STUDY POPULATION: Abdominal subcutaneous adipose tissue samples from $n=6$ healthy volunteers $(1 \mathrm{M}, 5 \mathrm{~F}$, age 28-38 y, BMI $24.5-63.0 \mathrm{~kg} / \mathrm{m} 2$ ) were collected by aspiration or during surgery. In 3 subjects, all females, paired femoral samples were also collected. After collagenase digestion approximately $n=10,000$ cells/ sample were used for single-cell RNA sequencing using the $10 \mathrm{X}$ Genomics platform. After QC and downstream analysis, data were analyzed in Seurat v.3.1.5. We identified first different cell types and then subclusters in an unbiased fashion. Gene Set Enrichment Analysis (GSEA) was used for pathway analysis. RESULTS/ 
ANTICIPATED RESULTS: Progenitor markers are related to extracellular matrix, eg DCN (logFC progenitors vs other cells types $=3.17$, expressed in $99.7 \%$ (pct.1) of progenitors, $26.1 \%$ (pct.2) of others). Endothelial and pericytes shared markers like RBP7 $(\operatorname{logFC}=1.67$, pct.1 0.88, pct.2 0.39); pericytes also showed unique markers, eg RGS5 ( $\log F C=2.29$, pct.1 0.89, pct.2 0.17). Progenitors are further divided into 11 sub-clusters, one of which showed enrichment of CD36 (high proliferation potential), FABP4 (differentiation), and of the novel marker PALMD $(\log F C=7.13$, pct.1 0.94, pct.2 0.48). All $\mathrm{p}<10 \mathrm{E}-5$. GSEA analysis suggests that inflammatory pathways are downregulated in both adipose progenitors and endothelial/pericyte cells in the femoral compared to the abdominal depot. DISCUSSION/SIGNIFICANCE OF FINDINGS: Single cell RNA sequencing provides unique insights into the molecular profile of cell types and the identification of novel subsets of cells within the human adipose tissue. Such cellular heterogeneity may explain differences in adipose function between individuals and eventually in the risk of obesity-associated metabolic diseases.

63438

Differential chromatin accessibility at dorsal root ganglia enhancers is associated with nerve injury

Kimberly E. Stephens ${ }^{1}$, Weiqiang Zhou ${ }^{2}$, Zhicheng $\mathrm{Ji}^{3}$, Hongkai $\mathrm{Ji}^{2}$, Yun Guan ${ }^{4}$ and Sean D. Taverna ${ }^{5}$

${ }^{1}$ Department of Pediatrics, University of Arkansas for Medical Sciences and the Arkansas Children's Research Institute, ${ }^{2}$ Department of Biostatistics, School of Public Health, Johns Hopkins University, ${ }^{3}$ Department of Biostatistics and Bioinformatics, Duke University, ${ }^{4}$ Departments of Anesthesia and Critical Care Medicine and Neurological Surgery, School of Medicine, Johns Hopkins University and ${ }^{5}$ Department of Pharmacology and Molecular Sciences, and the Center for Epigenetics, School of Medicine Johns Hopkins University

ABSTRACT IMPACT: Our improved understanding of the changes in chromatin accessibility that occur in persistent pain states may identify regulatory genomic elements that play essential roles in modulating gene expression in the DRG. OBJECTIVES/GOALS: Efforts to understand genetic variability involved in an individual's susceptibility to persistent pain support a role for upstream regulation by epigenetic mechanisms. Our objective was to examine the transcriptomic and epigenetic basis of persistent pain following nerve injury. METHODS/STUDY POPULATION: We used a multiomic approach to identify novel molecular pathways associated with nerve injury-induced pain hypersensitivity. Adult Sprague Dawley rats were randomized to Chronic Constriction Injury (CCI) to the sciatic nerve or no treatment (naive). The ipsilateral L4-L6 dorsal root ganglia (DRG)s were removed on Day 14 and used for ChIP-seq for H3K4me1, ATAC-seq, and RNA-seq. We assessed for differential chromatin accessibility, transcription factor motifs, and enrichment for biological processes in chromatin accessible regions associated with cis-regulatory regions identified by ATACseq and H3K4me1 enrichment. Luciferase assays determined the functional significance of these sequences. RESULTS/ ANTICIPATED RESULTS: We identified 58,446 genomic regions where H3K4mel enrichment overlapped with chromatin accessibility. Differential analysis identified 2145 of these 58,446 regions that had changes in accessibility after CCI. The majority of these regions were located in introns or intergenic regions. Functional annotation of the differentially accessible regions identified disparate molecular functions enriched following nerve injury which suggests that altered chromatin structure plays a role in the development of mechanical hypersensitivity. Motif analysis identified specific transcription factor families whose binding sequences were enriched in regions of increased or decreased accessibility. Luciferase assays showed significant enhancement or repression of gene transcription. DISCUSSION/SIGNIFICANCE OF FINDINGS: Our data provides a comprehensive map of chromatin accessibility changes in the DRG after CCI and emphasizes the importance of chromatin structure in the development and maintenance of chronic pain.

77232

The role of the bromodomain and extra-terminal motif (BET) family of proteins in head and neck cancer tumorigenic phenotype

Reilly A. Sample and Rachel Paolini

Washington University in St. Louis School of Medicine

ABSTRACT IMPACT: Understanding the biology underpinning head and neck cancer invasion and metastasis could lead to novel targeted therapies that are both effective and tolerable for patients with this debilitating disease. OBJECTIVES/GOALS: The bromodomain and extra-terminal (BET) family of epigenetic regulators has been implicated in the tumorigenesis of various cancers. In head and neck squamous cell carcinoma (HNSCC), the majority of morbidity is due to invasion and metastasis, so there is special interest in understanding the development of these phenotypes in HNSCC cells. METHODS/STUDY POPULATION: Stable SCC9 knockdown cell lines were generated by infecting cells with a lentivirus encoding Cas9-KRAB and a lentivirus encoding gRNA targeting each of the candidate BET proteins: BRD2, BRD3, BRD4, and BRDT. Knockdown was confirmed by qRT-PCR. Next, standard assays for proliferation (CellTiter-glo), invasion (Matrigel), and migration (scratch-wound healing assay) were performed for all candidate knockdowns and compared to a non-target control. RESULTS/ ANTICIPATED RESULTS: Proliferation assay results revealed that BRD4 knockdown had a significant negative effect on the proliferative capacity of SCC9 cells in vitro. Similarly, BRD4 knockdown SCC9 cell lines were less invasive and less migratory. Interestingly, knockdown of BRD2, BRD3, and BRDT had no effect on proliferation, invasiveness, or migration. DISCUSSION/SIGNIFICANCE OF FINDINGS: We have identified BRD4 as a key driver of the HNSCC tumorigenic phenotype. In the future, we plan to investigate the role of JQ1, a pan-BET inhibitor, on HNSCC cell phenotypes. Additionally, we will identify the downstream targets of BRD4, which may serve as potential therapeutic targets for both HNSCC as well as other cancers more broadly.

82125

Multiple epidemics of multidrug-resistant tuberculosis revealed by spatial disease mapping and whole-genome sequencing analysis in urban China

Chongguang Yang ${ }^{1}$, Yangyi Zhang ${ }^{2}$, Yuan Jiang ${ }^{2}$, Zheyuan $\mathrm{Wu}^{2}$, Xin Shen ${ }^{2}$, Joshua L. Warren ${ }^{1}$ and Ted Cohen ${ }^{1}$

${ }^{1}$ Yale University School of Public Health and ${ }^{2}$ Shanghai Municipal Center for Disease Control and Prevention

ABSTRACT IMPACT: Our study will integrate state-of-the-art methods in pathogen genomics, epidemiology, and geospatial analysis to identify both host- and pathogen-factors driving the MDR-TB transmission and the study outcome can inform the design of 